\title{
Las preocupaciones de docentes principiantes en contextos de
} ruralidad

The concerns of beginning teachers in rural contexts

\author{
Volumen 19, Número 1 \\ Enero-Abril \\ pp. $1-28$
}

Este número se publica el 1 de enero de 2019

DOI: DOI 10.15517/aie.v19i1.35407

Gerardo Ignacio Sánchez Sánchez

Ximena Elizabeth Jara Amigo

Revista indizada en REDALYC, SCIELO

Revista distribuida en las bases de datos:

LATINDEX, DOAJ, REDIB, IRESIE, CLASE, DIALNET, SHERPA/ROMEO, QUALIS-CAPES, MIAR

Revista registrada en los directorios:

ULRICH'S, $\underline{\text { REDIE}}, \underline{\text { RINACE}}, \underline{\text { OEI, MAESTROTECA, PREAL, }} \underline{\text { CLACSO }}$ 


\title{
Las preocupaciones de docentes principiantes en contextos de ruralidad

\author{
The concerns of beginning teachers in rural contexts
}

\section{Gerardo Ignacio Sánchez Sánchez ${ }^{1}$ Ximena Elizabeth Jara Amigo²}

\begin{abstract}
Resumen: El estudio tuvo por objetivo determinar y problematizar las preocupaciones que enfrenta la inserción laboral de personas egresadas de Pedagogía Primaria en condiciones culturales de ruralidad. Ello resulta importante, pues la incorporación de las nuevas personas docentes al campo laboral representa dificultades y desafíos que deben ser resueltos en un periodo de tiempo limitado si se pretende avanzar en el desarrollo profesional y evitar el abandono del ejercicio profesional. La investigación es cualitativa de tipo exploratoria descriptiva, basada en un diseño no experimental transeccional con administración de un cuestionario dirigido a cincuenta docentes principiantes en diversos contextos laborales rurales de la Región del Maule, Chile, y se profundiza en la problemática con el desarrollo de entrevistas semiestructuradas a ocho docentes en sus contextos de trabajo. Los resultados muestran que los contextos laborales de inserción condicionan (facilitan o limitan) el ingreso a la docencia y explican la presencia de preocupaciones de diversa naturaleza vinculadas a conocimiento disponible, a temores asociados y a dificultades de legitimidad que han debido superar. En conclusión, revelar estas preocupaciones resulta clave para aportar a los procesos de acompañamiento que en el contexto nacional comienzan a ser diseñados e implementados, y entender que su efectividad pasa por centrar el apoyo en el reconocimiento de la persona docente principiante como actor/actriz y no un simple factor del sistema.
\end{abstract}

Palabras clave: docentes principiantes, docencia, inserción profesional, contextos rurales.

\begin{abstract}
The objective of the study was to determine and problematize the worries that the labor insertion of graduates of Primary Pedagogy faces in cultural conditions of rurality. This is important, since the incorporation of the new teachers into the labour field represents difficulties and challenges that must be solved in a limited period, if we intend to advance in the professional development and avoid the abandonment of the Profession. The research is qualitative exploratory - descriptive, based on a non-experimental transectional design with administration of a questionnaire aimed at fifty beginning teachers in various rural labor contexts of the Maule Region, Chile, and deepens the problem with the development of semi-structured interviews to eight teachers in their work contexts. The results show that labor insertion contexts condition (facilitate or limit) the entrance to teaching, and explain the presence of worries of diverse nature linked to available knowledge, associated fears and difficulties of legitimacy that have had to overcome. In conclusion, unveiling these concerns is key to contributing to the accompaniment processes that begin to be designed and implemented in the national context, understanding that their effectiveness goes to focus the support on the recognition of the beginning teacher as an actor and not a simple factor of the system.
\end{abstract}

Key words: beginning teachers, teaching, professional insertion, rural contexts.

\footnotetext{
1 Académico de la Universidad Católica del Maule, adscrito al Departamento de Formación Inicial Escolar de la Facultad de Educación, Chile. Proyecto Interno Iniciación pedagógica de profesores de educación básica en contextos de ruralidad. Doctor en Ciencias de la Educación.Dirección electrónica: gsanchez@ucm.cl

${ }^{2}$ Académica de la Universidad Autónoma de Chile, de la carrera de Pedagogía en Historia, Geografía y Ciencias Sociales, Chile. Magister en Curriculum y Evaluación. Dirección electrónica:xjaraa@uautonoma.cl
}

Artículo recibido: 7 de junio, 2018

Enviado a corrección: 12 de setiembre, 2018

Aprobado: 29 de octubre, 2018 


\section{Introducción}

En Chile, la Ley 20.903 de Desarrollo Profesional Docente (2016), en su artículo 18, define la etapa de inducción al ejercicio profesional como proceso formativo que tiene por objeto acompañar y apoyar a la persona docente principiante en su ejercicio profesional para un aprendizaje, práctica y responsabilidad profesional efectivos. En ese marco legal, se diseña el Sistema Nacional de Inducción y Mentoría, con la intención de facilitar la inserción profesional e integración en la comunidad educativa a educadoras y docentes que inician su ejercicio profesional. Este derecho lo pueden ejercer quienes se encuentren en su primer o segundo año de ejercicio y se desempeñen en establecimientos subvencionados (municipales o particulares subvencionados).

La inducción es definida como un proceso formativo orientado a apoyar a docentes principiantes en el desarrollo de sus habilidades profesionales para su mejor desempeño; reducir el tiempo de adaptación al ejercicio profesional docente; apoyar la vinculación de la persona principiante con el establecimiento y el sistema local; y conectar la formación inicial con el desarrollo profesional.

En el contexto regional, la Universidad Católica del Maule enfrenta el desafío planteado por las políticas públicas para la formación docente de avanzar en la vinculación efectiva con el sistema escolar y de atender los requerimientos de los/las egresados/as en su entrada a la profesión. Es parte de los compromisos institucionales que la universidad asegure una permanente articulación con el sistema escolar, tanto por medio de la formación práctica como a través del desarrollo de las personas docentes que se incorporan al sistema, y de los establecimientos con los cuales se relacionan. Ello obliga a poner atención a los procesos de inserción a la docencia de las personas egresadas de pedagogía, explorando las dinámicas que el proceso asume en los diversos contextos socioculturales donde tiene lugar.

La Universidad Católica del Maule se sitúa en la región que se define como la más rural de Chile y con una alta vulnerabilidad económica y social (12,7\% sobre la media nacional de $8,6 \%$ según la encuesta CASEN) y es en estos contextos donde se insertan mayoritariamente las nuevas personas docentes, razón por la cual la ruralidad se configura en el escenario característico de ejercicio de la profesión.

Ruralidad que en la actualidad está adquiriendo otra fisonomía y que por tanto, no puede seguir siendo abordada en su distinción radical con lo urbano: aquellos regidos por fenómenos de carácter natural, con densidad poblacional baja, y con componentes culturales 
tradicionales que enfatizan la comunidad. En ese sentido, autores como Carneiro (2001) y Wanderley (2001) han adoptado la expresión de rururbano. Esta categoría supone que, en estos territorios, existe la mixtura campo/ciudad, la que se manifiesta en la condición de vida de algunos actores cuyos modos y lógicas de acción basados en valores, saberes y sentires rurales comienzan a mimetizarse con la urbe (y viceversa).

El artículo que se presenta, forma parte de un proyecto interno Iniciación pedagógica de profesores de educación básica en contextos de ruralidad en la Sub-Línea Regular Formación Docente. El objetivo que persigue ha sido explorar las diversas preocupaciones que la entrada al empleo y la profesión representa para personas docentes de Pedagogía Primaria egresadas de la Universidad Católica del Maule que se desenvuelven en escenarios geográficos rurales.

\section{Marco teórico}

\subsection{El proceso de inserción}

El proceso de inserción a la docencia supone enfrentar requerimientos profesionales y contextuales vinculados a una cultura escolar, y mediado desde las características personales de quienes lo desarrollan.

La investigación y las diversas Políticas Educacionales tendientes a la profesionalización de la docencia constatan que la formación inicial no asegura, de manera categórica, la apropiación de todas las herramientas básicas de la profesión, ya que muchas de ellas se adquieren durante los primeros años de enseñanza en el contexto de la práctica. Más aún, "los esquemas de actuación profesional se configuran mediante experiencias prácticas, preformativas y de formación inicial; cuya articulación se define durante la etapa de inicio a la enseñanza" (Fernández-Cruz, 2008, p. 68).

En ese sentido, la identidad profesional emerge como un proceso mediante el cual las personas docentes elaboran un concepto de sí en relación con su trabajo y profesión, presentándose como el conjunto de conocimientos, creencias, emociones e interpretaciones que se desarrollan a lo largo de la experiencia formativa y profesional (Ávalos, Cavada, Pardo y Sotomayor, 2010) los cuales en su conjunto deben permitirles comprender la cultura escolar dentro de los centros educativos, conectarse con ella de forma auténtica y reconocer las necesidades e intereses de familias y comunidades (Zeichner, 2010) mediante la construcción de relaciones de colaboración con la comunidad educativa. 
En la conquista de esta identidad emerge la etapa del desarrollo profesional conocida como inserción. La inserción profesional supone el proceso de transición desde el profesorado en formación hasta el profesional autónomo, como parte de un continuo desarrollo que se define por el (des) acople de tres variables: "las características de la formación inicial; las características de los contextos escolares donde se inserta el principiante y las formas de apoyo, y la presencia o ausencia de mentorías u otros apoyos que suavicen el proceso" (Ruffinelli, Cisternas y Córdoba, 2017, p. 18). En ese sentido, constituye "un periodo de tensiones y aprendizajes intensivos en contextos generalmente desconocidos y durante el cual los profesores principiantes deben adquirir conocimiento profesional además de conseguir mantener un cierto equilibrio personal" (Marcelo, 2009, p. 5).

Como periodo de transición de estudiante a docente, tradicionalmente se ha desatendido la complejidad que encierra esta etapa, y con ello creer que esa persona docente que se inicia en la tarea de enseñar a niños y jóvenes se conduzca en su trabajo como cualquier docente con experiencia, aun cuando la investigación constata que la entrada de principiantes en las aulas de clase como gestores y únicos responsables de un grupo de estudiantes se produce abruptamente. Por esta razón, la inducción profesional constituye "una fase crítica, por su intensidad y su concentración en un intervalo estrecho de tiempo" (Fernández-Cruz, 2008, p. 87). En ese intervalo, el itinerario de aprendizaje que sigue la persona docente principiante se sustenta en una visión situacional reducida de la tarea docente caracterizada por indicadores de comprensión limitada y de falta de perspectiva, que es preciso atender y acompañar.

Las experiencias de iniciación de las personas docentes durante sus primeros años se encuentran fuertemente condicionadas por los llamados macro y micro contextos.

Por una parte, los macro contextos, refieren a "las políticas educativas en torno a la profesión docente, el currículo y sus orientaciones, los sistemas de control y rendición de cuentas o la distribución segregada o equitativa dentro del sistema estudiantil con mayores niveles de vulnerabilidad" (Ruffinelli, Cisternas y Córdoba, 2017, p. 22).

Estos macro contextos demandan comprensión de los cambios sociales y culturales que inciden en el funcionamiento de las organizaciones escolares, en la definición de sus componentes culturales y finalmente, en las demandas al trabajo que debe desarrollar el profesorado. 
Por otra parte, se encuentran las demandas concretas planteadas por los micro contextos de inserción. Más específicamente, la adaptación a las "políticas idiosincráticas o locales asociadas al establecimiento escolar y su entorno: el modo de organizar la gestión del currículo, los espacios de competencia o colaboración entre docentes, la manera de concebir la enseñanza y el aprendizaje" (Ruffinelli, Cisternas y Córdoba, 2017, p. 22).

Enfrentado a la inserción, la evidencia muestra que:

Cuando están en una situación real de enseñanza, compleja, inestable, abierta a presiones de todo tipo y en la cual asumen por completo la responsabilidad de enseñanza, entonces entra en juego o se descubre otra forma distinta de formación de la que había visto hasta ese momento. (Baillauqués, 2010, pp. 74-75)

Específicamente, siguiendo al mismo autor, "el yo docente está desconcertado, piensa en los conocimientos y las habilidades que no tiene, que creía poseer o que no había presentado que fueran pertinentes, necesarios" (Baillauqués, 2010, p. 69).

Esta complejidad que enfrenta la persona docente en su inserción a la enseñanza se explica por diversas razones, una de las cuales la constituye su condición de principiante, y eso significa que cuando enfrenta el proceso de iniciación:

Tan sólo dispone de algunos proyectos conceptuales y, muy raramente, de los proyectores generados por la experiencia. El maestro novel, tiene, en consecuencia, una percepción parcial de la realidad y el más mínimo imprevisto es como un rayo luminoso que lo deslumbra e interfiere en su dominio de la situación. Tiene acceso a menos puntos de referencia para navegar y, con frecuencia, será demasiado tarde cuando perciba las señales que le sugieren cambiar de actitud o modificar un programa. (Carbonneau y Hétu, 2010, p. 123)

La llegada, por primera vez, de un joven profesor o profesora a una escuela se expresa en formas que no siempre son predecibles. La nueva persona docente puede ser acogida por la comunidad escolar e introducida formalmente a sus tareas, o simplemente ser dirigida a las aulas y a los niños/ niñas a quienes tendrá que enseñar. Puede significar que se les expliquen las reglas o rutinas del establecimiento o que las deba aprender a medida que se enfrenta con las consecuencias de no conocerlas. Puede encontrar un sistema institucionalizado de trabajo en conjunto al que debe integrarse en forma activa o pasiva, 
según si la edad y la experiencia son factores que se consideran respecto a esta participación. Ahora bien, este proceso está vinculado a lo que muchos autores "Ilaman "choque con la realidad", "choque de transición" e incluso "choque cultural", ideas que remiten a la confrontación inicial con la dura y compleja realidad del ejercicio de la profesión" (Tardif, 2004, pp. 61-62).

Lo cierto es que, aun cuando la inserción enfrenta desafíos comunes, las características personales de la persona docente principiante otorgan especificidad al proceso. Incluso, la inserción profesional es distinta según el contexto sociocultural al que se incorpore. La diferencia "puede deberse a la población estudiantil a la que se atiende, pero también a los recursos con los que dispone el establecimiento, o al modo de organización y de gestión que prevalece" (Ávalos, 2009, p. 46).

En ese sentido, la noción de que el profesorado ha cumplido la etapa de formación al salir de una institución universitaria es en la actualidad un supuesto que muchas políticas educativas comienzan a superar, respaldada en la constatación que ofrecen los estudios internacionales sobre la vida de las personas docentes y su inserción profesional. El profesorado, recién al comenzar su ejercicio profesional, entra en una etapa de aprendizaje docente que es clave para lo que será su futuro ejercicio. Los trabajos de Lortie (1975) y Grossman (1990), retomados por Ávalos, Carlson y Aylwin (2004), y actualizados por Ruffinelli, Cisternas y Córdoba (2017) muestran que este aprendizaje para muchos jóvenes docentes es un aprendizaje solitario sin ayudas especiales, y por tanto, centralmente, un aprendizaje de la experiencia.

El primer año se configura como un período de transición, en el que algunas personas docentes principiantes se sienten en un lugar desconocido al no pertenecer ya a la institución que las formó y por tanto, perder el carácter de estudiantes. Por otro lado, en la institución educativa que la acoge (o institución en general), la persona docente apenas está construyendo relaciones con la comunidad y haciendo su propio nicho en ella.

En consecuencia, la investigación y gran parte de las políticas educacionales en la actualidad, reconocen que la entrada a la profesión docente es necesaria de atender, pues en ese momento tiene lugar un proceso de aprendizaje que resulta crucial para el desarrollo y socialización profesional, el que requiere ser revisitado como acto de reconocimiento y de justicia social con el profesional. Sin olvidar que, en un escenario de crecientes demandas sociales y culturales, "la forma como cada docente novel enfrenta la enseñanza no es igual 
de un lugar geográfico a otro, ni dentro de sub-culturas de un mismo país. Tampoco lo es de un momento histórico a otro" (Ávalos, 2009, p. 44).

\subsection{Inserción a un contexto social y a una profesión}

La inserción docente incluye dos dimensiones. Por una parte, el proceso de búsqueda de empleo, que se espera sea estable o permanente; y por otra, la entrada a la profesión, que implica el periodo en que la persona docente principiante se incorpora a una cultura, y por esa razón "aprende a dominar su trabajo, a descubrir sus propios recursos y limitaciones, a constituirse un bagaje de conocimientos y habilidades que vienen de la experiencia misma de la profesión docente" (Tardif y Borges, 2013, p. 27).

Esta entrada a la profesión se relaciona con la tarea de construcción de identidad, la cual "no se forja en el vacío, sino en contextos concretos de experiencia y relación: se fragua, en definitiva, en el juego de unas transacciones sociales y biográficas que están sometidas a cambio y evolución" (Altarejos, Ibañez-Martín, Jordán y Jover, 1998, p.80).

La inserción debe ser comprendida como incorporación a un contexto sociocultural dinámico y complejo, y como entrada a una profesión que tiene sus especificidades. En el escenario actual, "el conjunto de las instituciones que caracterizaron la sociedad industrial están atravesando por profundas modificaciones, y que esto, a su vez muestra una sociedad que está reconfigurando sus dispositivos de control y reproducción social" (Tiramonti, 2004, p.19). Ello implicaría la existencia de una escuela que no logra disponer de una narrativa que le permita anclar la constitución de lo social (Duschatzky, 2001).

Al revisar las razones que explican este panorama, nos encontramos con la brecha que se produce entre la racionalidad que acompaña a la institución escolar y la naturaleza de las transformaciones sociales. "La creación y consolidación de los sistemas educativos nacionales modernos, junto con el surgimiento de instancias especializadas para la transmisión del oficio de enseñar conformaron una maquinaria de educar en masas" (Alliaud, 2017, p. 22) que persiguió la finalidad de "civilizar". De esa manera, la escuela se constituyó en un significativo agente de socialización, "una institución del orden, un dispositivo destinado a generar una comprensión compartida de la realidad" (Tiramonti, 2004, p.31), cuyo objetivo era preparar al individuo para la inserción a la vida social y cultural, llamada a promover de manera uniforme la preservación de la cultura, con un funcionamiento anclado a la burocracia planteada por Weber y a la lógica de la disciplina que trabaja Foucault. 
En la actualidad esta escuela se enfrenta a nuevas demandas que tensionan su legitimidad y que se encuentran asociadas a los procesos de globalización, la diversidad e inclusión, y las transformaciones en los procesos de socialización y construcción de sentidos. En consecuencia, los procesos de cambio "han puesto en crisis los viejos dispositivos escolares que contribuían a la producción de la autoridad pedagógica y al mantenimiento de un orden determinado que permitía el funcionamiento normal de las instituciones" (Tenti, 2007, p. 339).

Sin embargo, estas instituciones que mantienen cuestionada su legitimidad siguen funcionando y constituyen el contexto al que se incorpora la persona docente principiante. "En el caso de las escuelas, siguen siendo las únicas, por el momento, que permiten el pasaje del acervo cultural común entre las generaciones de modo sistemático y masivo" (Alliaud, 2017, p.24). Dejemos en claro que muchas de las dificultades que enfrentan estas instituciones que trabajan con otros derivan no solo de su especificidad, sino también de los nuevos órdenes sociales y culturales. En consecuencia, "la escuela se ha convertido en una institución sobredemandada y subdotada" (Tenti, 2004, p. 2).

Ahora bien, la persona principiante también se enfrenta a una profesión cuyas especificidades le generan demandas, no solo técnicas, sino también éticas y relacionales. De hecho, la profesionalización de la docencia, definida como imperativo de las diversas políticas educacionales, supone la consolidación de su dimensión cognoscitiva (referida al conjunto de conocimientos y destrezas que le faculten para el ejercicio de su trabajo) y éticodeontológica (vinculada al recto ejercicio de la tarea, en defensa de los derechos propios y de quienes a los cuales dirige su acción).

Desde esa perspectiva:

Todo educador, en lo que atañe a la dimensión cognoscitiva del ejercicio de su profesión, debe ocuparse de adquirir una serie de conocimientos relacionados con los contenidos - saber qué y para qué-; y, también debe conocer cómo llevar a cabo su trabajo - saber cómo-. Y por lo que respecta a la dimensión ética, conviene subrayar que aunque el ejercicio de cualquier profesión requiera asumir una responsabilidad moral, en el caso de los profesionales de la educación esta responsabilidad aumenta considerablemente, porque se trata de un trabajo que incide directa y profundamente en la vida de otros seres humanos. (García y García, 2012, p.174) 
Queda de manifiesto que "en todos los trabajos que se realizan de persona a persona (servicios personales) se exige el dominio de ciertas competencias técnicas instrumentales, más un plus ético de "compromiso", "respeto" y “cuidado por el otro" (Tenti, 2007, p. 349).

Asumir la tarea docente junto con requerir la adquisición de conocimientos y metodologías adecuadas para educar, cada vez mejor, implica el empeñarse en una labor de mejora personal —intelectual y moral—, más allá de los mínimos exigidos. Con el imperativo ético de no generar fabricación del ser humano, pues el objetivo de la empresa educativa es: "que aquél que llega al mundo sea acompañado al mundo y entre en conocimiento del mundo, que sea introducido en ese conocimiento por quienes le han precedido ... que sea introducido y no moldeado, ayudado y no fabricado" (Meirieu, 1998, p. 2).

Por tanto, y siguiendo la perspectiva de Tardif (2004), la condición de docente, supone una gran cantidad de expectativas que en el cumplimiento de su rol. Se espera que la persona docente transmita conocimientos, genere habilidades y capacidades en sus estudiantes. Además, hay expectativas de que cumplan un rol formativo importante. En esa medida, las personas docentes deben asumir funciones complementarias a las de la familia, la que para muchas de ellas es percibida como una sobrecarga.

Así, la persona docente se define como "un profesional de la articulación del proceso de enseñanza-aprendizaje en situación; un profesional de la interacción de las significaciones compartidas" (Altet, 2010, p. 38).

Ahora bien, al entender la enseñanza como posibilidad de obrar con otros, de formar, de transformar, de mediar, de colaborar en el aprendizaje de otros, constatamos la complejidad. Entre las razones, la fundamental es que niños y jóvenes:

Suelen resistirse a la intervención adulta y también muchas veces a la escuela; una escuela cuya institucionalidad o forma de imponer/socializar/educar se halla debilitada o resquebrajada; una escuela cuya forma predominante no condice con los modos de comunicación y socialización dominantes fuera de ella. (Alliaud, 2017, p. 30)

En ese sentido, el profesional docente ha de comprender que:

Lo "normal", en educación, es que la cosa «no funcione»: que el otro se resista, se esconda o se rebele. Lo «normal» es que la persona que se construye frente a nosotros no se deje llevar, o incluso se nos oponga, a veces, simplemente, para recordarnos que no es un objeto en construcción sino un sujeto que se construye. (Meirieu, 2003, p. 5) 
La persona docente se enfrenta a seres humanos individualizados y socializados al mismo tiempo, con los cuales desarrolla relaciones humanas de afectividad. En tanto seres individuales, las situaciones de trabajo se "refieren a situaciones muchas veces complejas, marcadas por la inestabilidad, por la unicidad, por la particularidad de los alumnos, que son obstáculos inherentes a toda generalización, a las recetas y a las técnicas definidas de forma definitiva" (Tardif, 2004, p.95). Asimismo, constituyen seres sociales con determinadas características socioculturales, de lo cual sufren innumerables influencias.

Por tal razón, el personal docente está llamado a hacer uso de su imaginación y de los recursos que tiene disponibles, además, de la voluntad y la motivación para desarrollar su trabajo. En definitiva, son sus propias cualidades personales las que marcan la diferencia entre el éxito o fracaso de la labor docente, más que los aprendizajes profesionales adquiridos.

El nuevo escenario social y las particularidades de la profesión:

Convierten a la enseñanza en un oficio que cada vez compromete más a la persona y que expone al individuo en tanto que individuo (y no en tanto que rol profesional). La inversión/inmersión personal en la tarea (trabajo concreto) puede ser gratificante, pero también puede ocasionar situaciones de depresión, en especial cuando no se cuentan con las competencias y condiciones de trabajo adecuadas y por lo tanto no se alcanzan los resultados esperados. (Tenti, 2007, p. 337)

En el caso particular de los nóveles, enfrentados o enfrentadas a las demandas de la enseñanza en el aula, se encuentran con dificultades que van sorteando como pueden ("se hunden o nadan"). Por tal razón, en su entrada a la profesión, inician un proceso de aprendizaje profesional que los ha de conectar con "unos juegos de reglas flexibles y cambiantes" (Carbonneau y Hétu, 2010, p. 123), para lograr distanciarse de la ilusión de la explicación teórica todopoderosa que en la práctica resultará siempre insuficiente.

A lo anterior, se suma que el trabajo docente en la actualidad ha cambiado su fisonomía, y con ello:

Enseñar hoy es ante todo crear, inventar, salirse del guión o del libreto. Probar y ser puesto a prueba en cada circunstancia. Aceptar y aprender a permanecer en la confusión. Para enseñar hoy necesitamos de saberes y habilidades que nos permitan probar, experimentar, re-crear y seguir aprendiendo continuamente. (Alliaud, 2017, pp. 31-32) 
En ese contexto, esta investigación sobre docentes principiantes se orienta a la indagación de los problemas que enfrentan estos en relación con la enseñanza, para develar sus principales preocupaciones en los contextos de inserción. En esa línea, obtener, por una parte, insumos que permitan retroalimentar el proceso de formación inicial, y por otra, sentar las bases para un acompañamiento efectivo a la iniciación pedagógica, proceso que viven generalmente en solitario, sin las ayudas necesarias que los vinculen tempranamente al ejercicio profesional y a una adecuada comprensión de la especificidad del trabajo docente.

\section{Metodología}

La investigación desarrollada es de tipo cualitativa, exploratoria-descriptiva, por lo tanto, inscrita en una perspectiva interpretativa (Pérez, 2007), la cual pretende relevar las nociones de comprensión, diálogo y acción, y tratar de resignificar el concepto de docente principiante como actor de su desarrollo profesional a partir de la recuperación de algunos insumos del paradigma de la narrativa en las ciencias sociales, el que busca comprender las realidades sociales desde las percepciones y significados de los sujetos que la configuran (Ruíz Olabuénaga, 2009).

\subsection{Tipo de diseño}

La investigación se fundamenta en un diseño no experimental de tipo transeccional descriptivo.

\subsection{Participantes}

La muestra asume un carácter no probabilístico. El universo total, para este caso, son las personas egresadas de la carrera de pedagogía en Educación Básica de la Universidad Católica del Maule. La población, para este estudio, corresponde a un subconjunto específico de estas personas: egresadas de las promociones 2015 y 2016, de las cuales emerge la muestra final que es de tipo intencional. Se define como criterio de inclusión para participar el que sean docentes de educación primaria; que se desempeñen en áreas rurales de la Región del Maule en Chile, que cumplan la condición de principiantes, y que manifiesten voluntad en participar.

De acuerdo con los criterios de inclusión definidos en el presente estudio, cincuenta docentes principiantes, vía firma de consentimiento decidieron libremente participar. Desde el 
punto de vista del tiempo de vinculación con la institucional actual, el 18\% lleva menos de un año y el $82 \%$ entre uno y dos años. En lo referido a la situación contractual, $82 \%$ declara trabajar a contrata y el 18\%, a través de SEP (Subvención Educacional Preferencial). Consultados por el tiempo de dedicación a la docencia, el 56\% lo hace en jornada completa de 44 hrs; el 18\%, en media jornada de 22 hrs; y el 26\% declara trabajar por hora. Respecto a las áreas disciplinarias en que se ejerce la docencia, las personas participantes imparten preferentemente las asignaturas de Educación Matemática, Lenguaje y Comunicación, Ciencias Naturales y Ciencias Sociales.

\subsection{Técnicas de recolección de información}

La investigación requirió desde el punto de vista instrumental dos aproximaciones. En un primer momento, y con una aproximación de carácter extensivo, que permitiera un primer levantamiento del estado de situación de la problemática, se administró un cuestionario tipo a 50 docentes noveles insertos en diversos contextos rurales de la región del Maule, Chile, vía online, y autoadministrado. El cuestionario explora las preocupaciones vinculadas al Marco para la Buena Enseñanza, entendido como instrumento técnico oficial que, a nivel de Chile, define las responsabilidades del personal docente en el desarrollo de su trabajo diario, con la finalidad de orientar las políticas de fortalecimiento de la profesión docente.

El cuestionario dispone de una escala que valora de 1 a 5 cada uno de los enunciados, donde 1 no representa ni ha representado ningún problema y 5 representa o ha representado un gran problema en su ejercicio docente. Es importante aclarar que, por problema o preocupación, entendemos que es una dificultad que encuentran las personas docentes principiantes en el desempeño de su tarea, de manera que los objetivos propuestos pueden verse limitados.

En un segundo momento, en una aproximación de carácter intensivo que da riqueza a la presente investigación, se visitó a 8 docentes en sus escuelas, con la intención de profundizar de manera situada las principales preocupaciones que se derivan de la relación actor - contexto. Para ello, se dispuso el uso de una entrevista semiestructurada, con guión de preguntas, previamente validado, que permitió obtener datos descriptivos al tiempo que facilitar un abordaje de tipo interpretativo por parte de los sujetos en torno a las preocupaciones y los condicionamientos presentados por los contextos de inserción. 


\subsection{Análisis de Resultados}

Se utilizó la técnica de análisis de contenido, la que permitió interpretar los datos recogidos e identificar categorías de análisis en los discursos de las personas participantes.

Según Ruíz Olabuénaga (2009), el proceso de análisis estableció tres operaciones importantes, a saber: una fase de reducción de datos en que se selecciona, simplifica y organiza la información para hacerla más abordable, y para ello se otorga a cada entrevista un código asociado a la inicial del nombre y un número correlativo; una fase de disposición y reducción de datos, a partir de registro categorial simple, que consideró el levantamiento de categorías de análisis (vinculadas a ámbitos de preocupaciones y condicionamientos ejercidos por los contextos de inserción), el que fue acompañado de la evidencia empírica que surgió de las entrevistas, y una fase final de obtención de resultados y conclusiones, en que se levantan nuevos elementos y explicaciones relacionadas con los objetivos de la investigación.

\section{Resultados}

Al finalizar la formación inicial, la persona docente enfrenta el desafío de la búsqueda de empleo y la entrada a la profesión, y por tanto se ve confrontada a una serie de situaciones vinculadas a la profesión y al contexto laboral, mediadas desde lo personal, en una tarea que incluye tensiones, dilemas, dudas y emociones.

Los resultados de la investigación se organizan en dos apartados: en una primera aproximación, las principales preocupaciones que evidencian personas docentes principiantes frente al proceso de enseñanza, y en una segunda, se profundiza cualitativamente en las preocupaciones en su relación con el contexto de inserción.

\subsection{Preocupaciones frente al proceso de Enseñanza}

El Marco para la Buena Enseñanza constituye el instrumento elaborado en el contexto Chileno que establece todas las responsabilidades de los y las docentes en el desarrollo de su trabajo diario, tanto las que asumen en el aula como en la escuela, y su comunidad.

Este instrumento nacional para el ejercicio profesional de los docentes organiza las funciones en cuatro dimensiones: preparación para la enseñanza, creación de un ambiente propicio para el aprendizaje, enseñanza para el aprendizaje de todo el estudiantado y las responsabilidades profesional. 
En la presente investigación se ha decidido indagar en torno a tres dimensiones del Marco para la Buena Enseñanza, y las preocupaciones de docentes principiantes muestran los siguientes resultados.

\subsubsection{Preparación de la enseñanza}

Esta dimensión del Marco para la Buena Enseñanza está referida, fundamentalmente, a las competencias pedagógicas necesarias para organizar el proceso de enseñanza. Este proceso requiere atender las necesidades estudiantiles y los requerimientos de los contextos socioculturales (Ver Tabla 1).

Tabla 1

Porcentaje de docentes principiantes que indican preocupaciones asociadas con la preparación de la enseñanza, según Marco para la Buena Enseñanza, 2017

Preocupaciones

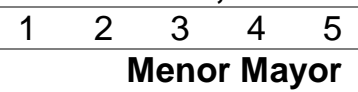
Dificultad

Diseñar situaciones realmente desafiantes

Planificar para la heterogeneidad del estudiantado

$\begin{array}{lllll}8 & 26 & 36 & 20 & 10\end{array}$

Manejar diferentes tipos de planificaciones (estructura)

Lograr coherencia objetivos-actividades-evaluaciones

Planificar sobre contenidos desconocidos

$\begin{array}{lllll}12 & 24 & 36 & 20 & 8\end{array}$

$\begin{array}{lllll}32 & 28 & 22 & 12 & 6\end{array}$

Fuente: Elaboración propia de los autores (2017), con información del Marco para la Buena Enseñanza (2008).

Esta dimensión muestra fuentes de preocupación para las personas participantes en lo referido a elaborar diseños de aula, que logren responder a las necesidades del estudiantado y atender a la diversidad presente en el aula. Sin embargo, aparecen como aspectos menos preocupantes y probablemente mejor resueltos los concernientes al manejo de distintos tipos de planificación y el logro de coherencia entre objetivos declarados - actividades de aprendizaje y procedimientos de evaluación.

\subsubsection{Preocupaciones vinculadas a la enseñanza}

Esta dimensión hace referencia al entorno de aprendizaje en su sentido más amplio, así como a los aspectos involucrados en el proceso de enseñanza gestionados por el profesorado en pos del aprendizaje de sus estudiantes (ver Tabla 2). 
Tabla 2

Porcentaje de docentes principiantes que indican preocupaciones asociadas al desarrollo de la enseñanza, según Marco para la Buena Enseñanza, 2017

\begin{tabular}{lrrrrr}
\hline \multirow{2}{*}{\begin{tabular}{l} 
Preocupaciones \\
\cline { 2 - 6 }
\end{tabular}} & \multicolumn{5}{c}{$\begin{array}{c}\text { Menor Mayor } \\
\text { Dificultad }\end{array}$} \\
\hline Enseñar lo que no se maneja & 10 & 28 & 26 & 26 & 10 \\
\hline Enseñanza que responda a la heterogeneidad & 6 & 34 & 30 & 22 & 8 \\
\hline Gestionar tiempos & 8 & 38 & 26 & 24 & 4 \\
\hline Aprovechar el error como instancia de aprendizaje & 52 & 16 & 24 & 4 & 4 \\
\hline Capturar y mantener la atención en clases & 22 & 32 & 26 & 18 & 2 \\
\hline ¿Cómo enseño un contenido? & 10 & 40 & 26 & 18 & 6 \\
\hline Contar con distintas formas para enseñar un contenido & 16 & 36 & 26 & 14 & 8
\end{tabular}

Fuente: Elaboración propia de los autores (2017), con información del Marco para la Buena Enseñanza (2008).

En esta dimensión se muestran, particularmente problemáticas, la tarea de trabajar temas o contenidos que no se dominan, y particularmente, el desarrollar una enseñanza que logre responder a la heterogeneidad que muestra el estudiantado. También constituye una preocupación para la persona docente el lograr una adecuada gestión de los tiempos del aula. Asimismo, aparece como aspecto menos problemático de la enseñanza el reconocer y aprovechar el error como fuente de aprendizaje.

\subsubsection{Preocupaciones vinculadas a la evaluación}

Esta dimensión refiere las competencias de la persona docente asociadas a la evaluación, y por consiguiente a sus posibilidades de monitorear, en forma permanente, los aprendizajes y necesidades detectadas en sus estudiantes (ver Tabla 3).

Tabla 3

Porcentaje de docentes principiantes que indican preocupaciones asociadas al desarrollo de la evaluación, según Marco para la Buena Enseñanza, 2017

\begin{tabular}{|c|c|c|c|c|c|}
\hline \multirow[b]{2}{*}{ Preocupaciones } & \multirow{2}{*}{\multicolumn{5}{|c|}{$\begin{array}{cccc}1 & 2 & 3 & 4 \\
\begin{array}{c}\text { Menor Mayor } \\
\text { Dificultad }\end{array}\end{array}$}} \\
\hline & & & & & \\
\hline ¿Cómo evalúo el proceso? & 16 & 26 & 28 & 24 & 6 \\
\hline Evaluar diferenciadamente & 14 & 28 & 26 & 28 & 4 \\
\hline Evaluar habilidades y no solo conocimientos & 8 & 28 & 30 & 26 & 8 \\
\hline Ajustar evaluación a lo específico de cada subsector & 26 & 26 & 22 & 22 & 4 \\
\hline mentos (prue & 34 & 8 & 22 & 14 & 2 \\
\hline
\end{tabular}

Fuente: Elaboración propia de los autores (2017), con información del Marco para la Buena Enseñanza (2008). 
Esta área del trabajo docente constituye una fuente de preocupación e inseguridad profesional para la persona principiante, particularmente en lo referido a cómo se evalúa y atiende al proceso de enseñanza y aprendizaje, el desarrollar evaluación diferenciada y lograr evaluación no solo de conocimiento factual y conceptual, sino fundamentalmente de habilidades.

Ahora bien, las preocupaciones que evidencian este grupo de docentes principiantes (Marcelo, 2008), nóveles (Imbernón, 2007), novatos (Conderman y Johnston-Rodríguez, 2009) o debutantes (Cornejo, 1999) en relación con los requerimientos planteados por el Marco para la Buena Enseñanza (2008), ponen de manifiesto la complejidad de los procesos de enseñanza y aprendizaje. Vale decir, emergen necesidades, inquietudes y problemáticas que ponen de manifiesto la especificad de la profesión y el trabajo docente, dado su carácter interactivo y relacional que implica un fuerte compromiso personal (Tardif, 2004; Davini, 1995; García y García, 2012).

Específicamente, este componente del trabajo docente es altamente reconocido por los docentes principiantes consultados, para quienes el diseñar situaciones de trabajo que representen un desafío cognitivo y afectivo para el estudiantado con el que se trabaja; el planificar en pos de la heterogeneidad que, en términos de ritmos y estilos de aprendizaje, evidencian los sujetos; y luego generar una enseñanza que atienda efectivamente a la heterogeneidad, así como el gestionar adecuadamente los tiempos de aula, terminan por configurar un conjunto de preocupaciones que se relacionan con el carácter distintivo de la profesión y la tarea, como bien lo reportan Tardif (2004), García y García (2012), Meirieu (2003), entre otros.

Complementariamente, la evaluación del aprendizaje se perfila como un ámbito clave de inquietudes y de tareas que es preciso aprender a dominar, pues, en sus planteamientos, el hacer efectiva la evaluación del proceso, el lograr evaluar de manera diferenciada y el superar la evaluación de conocimiento, para avanzar a las habilidades, constituyen aspectos de la práctica docente que no están dominados.

Estas preocupaciones resultan altamente coincidentes con los desafíos que encierra el ingreso a la profesión (Tardif y Borges, 2013).

No obstante, este ingreso o entrada a la docencia no puede ser comprendido al margen de los contextos de inserción, máxime, cuando, en el escenario chileno actual, la existencia de una Política de Desarrollo Profesional Docente (2016) viene a desarrollar 
nuevas comprensiones y mecanismos de apoyo para las personas docentes que se inician profesionalmente en espacios educativos diversos. De hecho, esta política reconoce en su fundamentación la existencia a nivel país de una realidad nacional, socioeconómica y culturalmente segregada, que es el contexto donde este docente se ve movilizado a vivir una etapa de aprendizaje docente crítica para el desarrollo profesional y de construcción de su identidad (Ávalos, Carlson y Aylwin, 2004; Marcelo, 2009).

\subsection{Preocupaciones derivadas de los contextos de inserción}

\subsubsection{Descripción de la escuela como espacio de inserción}

La escuela aparece como el espacio natural donde se desarrolla la inserción y actúa como un espacio condicionante del trabajo docente, vale decir, en algunos casos, facilita la temprana iniciación de la persona principiante, y en otros, dificulta la incorporación al ejercicio de la profesión.

En el primer caso, la escuela se configura como un espacio que favorece la inserción docente cuando, en ella, — desde la perspectiva de los principiantes - se visualizan elementos tales como:

[...] Una escuela en constante cambio... con docentes muy comprometidos y con altas expectativas de los alumnos y con la convicción que todos deben aprender, siempre preocupados de trabajar con la inclusión (E2)

[...] Una escuela inserta y arraigada en patrones culturales campesinos y agrícolas con miras a hacer conexión con el mundo globalizado (E4)

[...] Una comunidad educativa diversa, muy inclusiva en todo aspecto, donde se le da cabida a cualquier persona, sin importar su pasado, dificultades, raza, género o si tiene consigo familia o no (E5)

[...] Una escuela donde los profesores tienen buenas relaciones pese a lo difícil que resulta sacar adelante a los niños (E6)

En estos casos, las escuelas a las que se insertan a trabajar constituyen espacios positivos en los cuales logran identificar oportunidades profesionales y laborales, dentro de las cuales destacan:

[...] Capacidad de trabajar con la diversidad social, cognitiva y cultural, desarrollando una docencia pertinente a las necesidades de los niños (E5) 
[...] Atender las particularidades del contexto rural, evidenciando compromiso del profesorado con altas expectativas sobre el alumnado y sus posibilidades de aprendizaje (E2)

[...] Atender las necesidades formativas en contextos vulnerables, sensibles a la comunidad, trabajar colaborativamente aportando ideas, en una organización democrática (E4)

En forma natural y paralela, las escuelas también desarrollan culturas o prácticas que entorpecen la inserción, particularmente, cuando se trata de espacios con prácticas tales como:

[...] Una escuela carente de la formación humana y valores para nuestros estudiantes donde su único papel es tratar de lograr algún día buenos resultados SIMCE (E7).

[...] Una escuela tensionada entre el desafío de lograr aprendizajes y las complejidades de un medio adverso y que ofrece indiferencia y oposición a la tarea del profesorado. La mayor parte de las veces una escuela cerrada, que no se proyecta en sus actividades a la comunidad en general (E8)

[...] Una escuela donde los directivos han cedido a las complejidades del contexto, justificando en su vulnerabilidad la decisión de no asumir compromisos con el aprendizaje (E3)

[...] Una escuela donde el individualismo no permite aunar esfuerzos para hacer frente a una tarea cada vez más difícil con carencias de todo tipo: afectiva, emocional, social, y sin posibilidades de mejorar (E1)

Desde su perspectiva, la existencia de escuelas con esas culturas internas representa importantes fuentes de amenaza a la inserción y al desarrollo de un trabajo docente de calidad. Específicamente, los relatos refieren a:

[...]Establecimiento visto como guardería por padres y apoderados, con poco compromiso frente al aprendizaje de sus hijos (E8)

[...]Cultura escolar no centrada en los estudiantes con escasas expectativas frente a los estudiantes. La vulnerabilidad del contexto y la escuela, hace difícil trabajar con este tipo de estudiantes (E3)

[...]Muchas demandas administrativas que disminuyen los tiempos para el aula y la atención a las necesidades de los alumnos (E7) 
[...]Complejidad del clima laboral asociado a falta de claridad respecto al proyecto institucional del establecimiento y la presencia de nepotismo de los directivos en sus decisiones (E1)

\subsubsection{Descripción de las preocupaciones que experimentan docentes principiantes}

Según se advierte en la indagación en cuanto a las preocupaciones manifestadas por las personas docentes principiantes en su inserción, los relatos permiten configurar categorías de análisis en torno a: "sensación de no saber todo lo necesario"; "temores que genera la docencia"; "legitimidad frente al estudiantado"; "compleja relación con las personas discentes"; y "dinámicas que presentan los contextos de inserción".

Respecto a la primera categoría: "sensación de no saber todo lo necesario para desarrollar el trabajo docente", los sujetos ponen de manifiesto situaciones vinculadas a:

[...] No contar con todos los conocimientos y herramientas para desarrollar mi rol como profesor, considerando que en el aula siempre pasan situaciones que es preciso afrontar (E2)

[...] Necesidad de entender los lineamientos técnicos del establecimiento para evitar errores (E4)

[...] Conocimiento profundo de quienes son y como aprenden sus estudiantes para asegurar que lo planificado genere entusiasmo e interés de los alumnos (E6)

[...] Herramientas para atender los diversos requerimientos administrativos y de las familias para las cuales considero que no fui suficientemente preparado en la universidad (E1)

[...] No disponer de todas las estrategias para lograr que todos sus estudiantes aprendan, dada la diversidad presente en las aulas y que superan con creces lo que uno pudiera imaginar o suponer (E8)

En lo referido a la segunda categoría que emerge: "temores que genera la docencia", sus relatos articulan expresiones de miedo o inseguridad asociadas a:

[...] Reacciones muchas veces inesperadas de los estudiantes con necesidades derivadas de los contextos familiares que no sé cómo afrontar (E6)

[...] Alta vulnerabilidad de los niños y sus familias que hacen difícil avanzar en los aprendizajes de las distintas asignaturas (E3) 
[...] Temor para enfrentar a familias que en general llegan a la escuela a pelear y siempre a la defensiva haciendo difícil lograr una comunicación efectiva (E8)

[...] Temor a bajar los esfuerzos como ya lo hicieron muchos profesores y no tener expectativas respecto a lo que los alumnos puedan ser capaces de aprender (E3)

[...] En muchas ocasiones he sentido miedo de no ser capaz de desarrollar actividades innovadoras, entretenidas que motiven a los estudiantes y que logren responder a sus intereses, sus estilos de aprendizaje (E5)

En lo relacionado con la tercera categoría: "legitimidad frente al estudiantado", los principiantes advierten con matices diversos, situaciones como las siguientes:

[...] Muchas veces uno observa actitudes irrespetuosas de algunos estudiantes con uno y con lo que les intento enseñar y eso me preocupa pues uno intenta hacer lo mejor posible (E5)

[...] Las normas de convivencia en el aula hay que estar recordándola siempre, pues los niños son desordenados, se molestan con facilidad y se amurran. A la mayoría les cuesta respetar normas y eso me complica pues tiendo a perder mucho tiempo en la sala y al final me desgasto (E2)

[...] Temor a no lograr el respeto de los estudiantes y sus familias, no tener dominio de grupo me complica pues pienso que a lo mejor me falta carácter o ser más firme (E7)

[...] La indisciplina de los alumnos en segundo ciclo es un problema que tenemos los profesores, pues a los niños todo les aburre, se distraen con facilidad y quieren cosas entretenidas porque o sino comienzan con el desorden (E6)

[...] Me ha costado y de hecho creo que todavía no lo he logrado el ganarme el respeto de mis estudiantes pues los alumnos se dan cuenta que en la escuela el cumplimiento de las normas es escaso y por lo tanto a uno le falta más apoyo de la escuela en ese sentido (E3)

Por lo mismo, y particularmente en el segundo ciclo de la educación básica, emerge la cuarta categoría: "relación con el cuerpo discente". Desde su perspectiva, ello se traduce en preocupaciones vinculadas a:

[...] Atender las necesidades valóricas de los alumnos es difícil pues las familias están poco presentes en el aprendizaje, de hecho cuando vienen a la escuela es a reclamar y siempre justificando situaciones (E7) 
[...] Ocupo mucho tiempo en el aula intentando crear un clima de respeto entre los estudiantes y es un tema de nunca acabar, pues los niños están muy inquietos (E2)

[...] Dificultad para manejar a ciertos estudiantes que tienden a hacer difícil la disciplina en la sala y que mueven a otros al desorden (E6)

[...] En muchas ocasiones me doy cuenta que no trato a todos mis alumnos de la misma manera y me siento culpable, aunque sea de manera inconsciente (E8)

[...] La relación con los alumnos complicados resulta más difícil cuando no se tiene el apoyo de la familia y especialmente de la misma escuela (E3)

Finalmente, en lo que refiere a la quinta categoría: "los contextos de inserción y las dinámicas culturales de las escuelas", las inquietudes se centran en:

[...] Existencia de una escuela que no tiene muchas expectativas de que los niños por ser de campo puedan salir adelante y por lo mismo se conforman con lo mínimo (E3)

[...] Lo más complicado han sido los colegas y sus formas, pues con los niños no es tan difícil la cosa, en cambio me agota tener que lidiar con profesores que siempre están descontentos y encuentran todo mal en el resto, que los alumnos son flojos, que las familias no están ni ahí, que los jefes no apoyan y siempre tirando para abajo lo que uno quiere hacer (E7)

[...] Me preocupó mucho en su momento no saber todas las rutinas administrativas como profesor pues sentía que los demás esperaban que las manejara, y uno no quiere cometer errores pues es su trabajo y quería hacerlo bien (E4)

[...] No tengo claridad cómo enfrentar a las familias, pues permanentemente en la escuela a uno le dicen que las familias son complicadas y que mejor no meterse con ellas, pero uno se da cuenta que las necesita para lograr que los niños aprendan (E3) [...] Lo que más me costó fue enfrentar muchos requerimientos, tales como la planificación, las pruebas, las entrevistas con apoderados y los informes de rendimiento y tener que resolverlos en poco tiempo y tratando de no cometer errores, pues habitualmente es poco el apoyo que se recibe. Durante el primer año uno todo lo considera grave, con el tiempo he ido aprendiendo que no todo tiene la misma urgencia (E1) 
Una entrada en la docencia que no sea satisfactoria para el profesional principiante tiene consecuencias tanto para el propio proceso de desarrollo profesional como para las instituciones educativas y sus estudiantes.

Entre la búsqueda de empleo y el ingreso a la profesión, este grupo de principiantes investigados manifiesta una serie de preocupaciones altamente interrelacionadas, poniendo de manifiesto el carácter complejo que muestra el ejercicio de la docencia, y en ese sentido "los problemas con los que se enfrentan los profesores en las escuelas no suelen responder a un área del conocimiento en particular, a una porción curricular o a un curso" (Alliaud, 2017, p. 96) Los problemas propios del "pantano" (al decir de Schon) son complejos y poco definidos, y para enfrentarlos se requiere necesariamente un aprendizaje desde la experiencia docente misma en situaciones fluidas, cambiantes y crecientemente inciertas. Enfrentado a ese desafío, el principiante inicia un abrupto aprendizaje, reconociendo que para "cuando se produce la inmersión en las aguas revueltas de la escuela, de poco sirve el conocimiento acumulado durante la formación inicial" (Fernández-Cruz, 2008, p. 86).

Analizado en una perspectiva de desarrollo, la inducción profesional configura una fase crítica, por su identidad y su concentración en un intervalo estrecho de tiempo, resultando ser un momento en el que se agudizan las necesidades formativas docentes y para el que tradicional y paradojalmente no han existido apoyos institucionalizados en nuestro contexto.

Las necesidades de esta etapa del desarrollo profesional se justifican en la medida que el principiante, en pos del conocimiento experto, evidencia una actuación caracterizada por guías de acción basadas sobre atributos o aspectos; percepciones sobre situaciones aún limitadas; y donde todos los atributos y aspectos de la realidad que enfrenta son tratados separadamente, y a ellos otorga la misma importancia.

Hablar de inducción o inserción es aludir a la interrelación de dos grandes ámbitos de la realidad: la realidad psicobiológica, que representa el sujeto y la realidad sociocultural en que descansa la primera. La inducción se desenvuelve en estos dos ámbitos claves: el sujeto (entidad psicobiológica) y el espacio sociocultural (representado por la escuela).

De esa manera, el proceso de inserción a la docencia se realiza en situaciones sociales, las cuales constituyen concreciones en el espacio y el tiempo de la estructura social (ámbito objetivo) percibidas y vivenciadas por el sujeto (ámbito subjetivo), y que se resuelven en la interrelación de estos ámbitos para constituir un repertorio de posibilidades limitaciones formativas para la persona docente principiante. 
Figura 1

Situaciones sociales que condicionan la inserción

\section{SITUACIÓN POSIBILITANTE}

Gestión directiva con centralidad en el estudiantado y su aprendizaje Apertura a la diversidad e inclusión

Existencia de espacios de trabajo profesional colaborativos

Escuela en sintonía con los contextos socioculturales

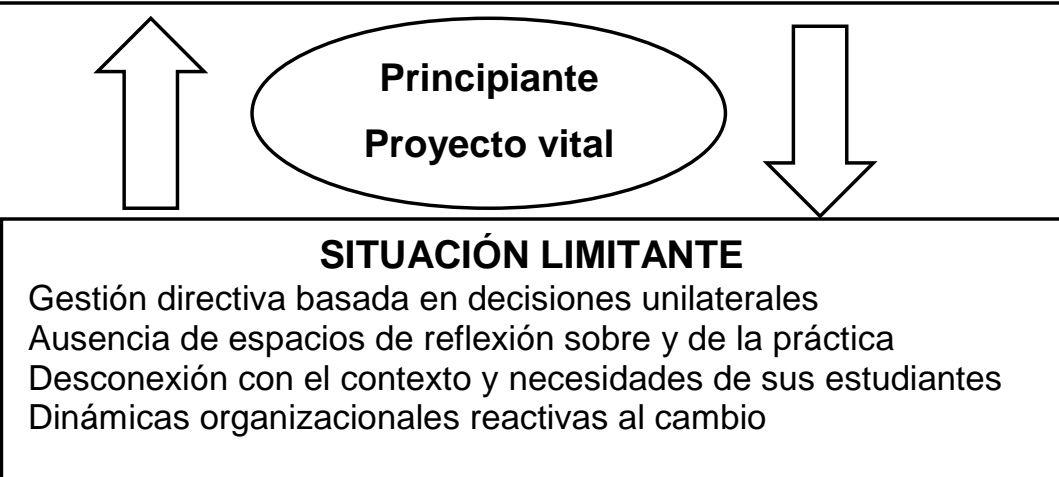

Fuente: Elaboración propia de los autores (2017)

En esa perspectiva, la investigación reporta que las escuelas en cuanto espacio natural donde se realiza la inserción, actúan como marco sociocultural condicionador de la inserción, vale decir, nos encontramos con aquellas que configuran situaciones sociales posibilitantes, y otras, que juegan el rol de situación social limitante. En el primer caso, los sujetos aluden a escuelas representadas como espacios de potencialidad y crecimiento con esfuerzos institucionales puestos en el aprendizaje del estudiantado, apertura a la diversidad e inclusión, instituciones capaces de articular el trabajo colaborativo del profesorado y equipos directivos, capacidad de sintonizar con el contexto y las necesidades del estudiantado como foco de la gestión desarrollada, entre otras.

En el segundo caso, emergen escuelas descritas como espacios de trabajo donde las decisiones son unilaterales, y por tanto, no existe posibilidad de disenso o crítica, instituciones desconectadas del contexto y de sus estudiantes, escuelas que han renunciado a su tarea inicial, justificadas en la condición de vulnerabilidad o espacios donde las dinámicas internas llevan a un permanente comienzo de proyectos e iniciativas, sin asegurar condiciones para su implementación y monitoreo.

En ambos casos, queda de manifiesto, que los "distintos modos de organizarse en los establecimientos educativos generan diferentes culturas profesionales y formas de enfrentar la labor cotidiana" (Ruffinelli, Cisternas y Córdoba, 2017, p.23). No obstante, todas estas 
expresiones con las cuales se describe a las escuelas refieren la complejidad cultural de la institución escolar, planteando al principiante demandas que se presentan en un repertorio de posibilidades (oportunidades) o limitaciones formativas (debilidades) en torno a lo cual se definen los procesos de iniciación profesional.

En ese marco de complejidad, es posible entender mejor las preocupaciones del personal docente principiante, las que se bien fijan en la relación con el estudiantado una de sus principales fuentes de desconcierto, desbordan el espacio del aula y se amplían a las problemáticas del contexto escolar y las relaciones con la comunidad. En cuanto a la relación con el estudiantado, las dificultades se expresan en ansiedades y sensaciones de la persona novel de que existirían aspectos de la enseñanza que permanecen aún inaccesibles y vedados para ella. A lo anterior, se agrega la presencia de temores asociados a no estar a la altura de las circunstancias y de demandas del aula, lo que queda reflejado en la dificultad para validar la legitimidad de la palabra de la personal docente y con ello, asumir la investidura docente en su relación con el estudiantado.

Como parte de las preocupaciones fuera del aula, encontramos inquietudes e impaciencias referidas a las dinámicas de los contextos escolares, las cuales, reflejadas en las expresiones de incertidumbre y vulnerabilidad, terminan acechando a la persona docente, pues, resulta difícil la tarea de enseñar cuando las demandas sobre la profesión no son claras y las certezas tienden a flaquear.

Estas preocupaciones ponen de manifiesto la necesidad de una formación inicial y un acompañamiento que articule en un continuo el proceso de desarrollo profesional, anticipando mejores espacios formativos, ya que "los docentes tendrán más chance de operar/obrar/enseñar, es decir, ejercer su oficio, en situaciones fluidas, cambiantes, complejas e inciertas, si se los prepara para hacerlo (Alliaud, 2017, p. 97)

\section{Conclusiones}

Los resultados de la presente investigación permiten concluir que la persona docente se enfrenta a la complejidad de un proceso de iniciación durante sus primeros años, que se encuentra condicionado no solo por lo que ocurre fuera del aula, y constituye el contexto macro social, sino que también se muestra fuertemente influenciado por la adaptación a las dinámicas propias del micro contexto de las escuelas, como espacios donde se resuelve el proceso de desarrollo profesional. En una dinámica relación estructura y agencia, 
observamos la presencia de contextos de inserción que condicionan (facilitan o limitan) el ingreso a la docencia, y que permiten comprender desde una perspectiva individual 0 personal, una serie de preocupaciones de diversa naturaleza (vinculadas al conocimiento, a temores, a legitimidad, a relaciones y contextos) que en definitiva han de afrontar.

En esa perspectiva, la investigación aporta antecedentes valiosos para retroalimentar los procesos formativos al interior de la institución universitaria y contribuir, además, a orientar el desarrollo profesional docente.

Respecto a la institución formadora, y con la intención de favorecer los procesos de acreditación obligatoria para las carreras de pedagogía, el vincular la formación inicial con el desarrollo profesional docente aporta a la comprensión del carácter complejo de la docencia en contextos reales, lo cual favorece el desempeño profesional a partir de una relación dialéctica, teórica y práctica que permite vivenciar la teoría y fundamentar la práctica.

En lo referido al desarrollo profesional, el conocimiento de la complejidad que presenta la etapa de iniciación pedagógica, a la cual pretende responder la Ley 20.903 (2016) en implementación, ha de permitir la generación de espacios para acompañar, apoyar y facilitar la inserción e integración de docentes principiantes, según las necesidades que experimentan y las preocupaciones que emergen en estrecha sintonía con los contextos.

La clave de estos acompañamientos pasa, necesariamente, por centrar el apoyo en el reconocimiento de la persona docente principiante como protagonista, y no como un simple factor del sistema. De tal manera que sea posible un verdadero proceso de desarrollo profesional. De hecho:

Cuando el profesor o profesora se incorpora por primera vez al trabajo en un centro escolar, lo hace con la ilusión y preocupación lógica en un principiante, generalmente joven, y durante un período no desdeñable su situación y actividad profesional están trufadas del deseo o la necesidad de ser aceptados, de asimilar lo que hacen los compañeros, de aplicar lo aprendido durante la formación inicial, también por la inevitable reproducción de pautas aprendidas durante su propia escolaridad, todo ello mezclado con el temor a no controlar la clase (la disciplina, la atención a demandas y necesidades diversas) y cumplir la programación. (Vélaz de Medrano, 2009, p. 210)

Se hace necesario reconocer que la inserción a la docencia se desarrolla en un contexto y en interacción con sujetos. Por consiguiente, no puede ser comprendida al 
margen de los contextos escolares y socioculturales, pues sus particularidades -en nuestro caso una región con altos índices de ruralidad y vulnerabilidad social y económicacondicionan finalmente el ingreso a la docencia y la profesión de quienes serán docentes que siguen estando una y otra vez en el centro de la escena pedagógica. De ahí la necesidad de seguir profundizando en su estudio.

\section{Referencias}

Altarejos, Francisco, Ibañez-Martín, José, Jordán, José y Jover, Gonzalo. (1998). Ética docente. Barcelona, España: Ariel.

Altet, Marguerite. (2010). La competencia del maestro profesional o la importancia de saber analizar las prácticas. En Leopold Paquay, Marguerite Altet, Evelyne Charlier y Philippe Perrenoud (Coords.), La formación profesional del maestro (pp. 33-54). D.F., México: FCE.

Alliaud, Andrea. (2017). Los artesanos de la enseñanza. Buenos Aires, Argentina: Paidós.

Ávalos, Beatrice, Cavada, Paula, Pardo, Marcela y Sotomayor, Carmen. (2010). La profesión docente: temas y discusiones en la literatura internacional. Estudios Pedagógicos, 36(1), 235-263.

Ávalos, Beatrice. (2009). La inserción profesional de los docentes. Profesorado. Revista de Currículum y Formación de Profesorado, 13(1), 43-59.

Ávalos, Beatrice, Carlson, Beverly y Aylwin, Pilar. (2004). La inserción de profesores neófitos en el sistema educativo: ¿cuánto sienten que saben y cómo perciben su capacidad docente en relación con las tareas de enseñanza asignadas? (Proyecto Fondecyt 1020218). Santiago, Chile: Comisión Nacional de Investigación Científica y Tecnológica (Conicyt).

Baillauqués, Simone. (2010). El trabajo de las representaciones en la formación de los maestros. En Leopold Paquay, Marguerite Altet, Evelyne Charlier y Philippe Perrenoud (Coords.), La formación profesional del maestro (pp. 55-87). D.F., México: FCE.

Carbonneau, Michel y Hétu, Jean Claude. (2010). La formación práctica de los maestros y el nacimiento de una inteligencia profesional. En Leopold Paquay, Marguerite Altet, Evelyne Charlier y Philippe Perrenoud (Coords.), La formación profesional del maestro (pp. 107-138). D.F., México: FCE.

Carneiro, María José. (2001) ¿Do rural e do urbano: uma nova terminología para una velha dicotomía ou a reemergencia da ruralidade? En II Seminario sobre: o Novo Rural Brasileiro. Campinas, Brasil: IE/Unicamp.

Conderman, Greg, y Johnston-Rodriguez, Sarah. (2009). Beginning Teachers' Views of Their Collaborative Roles. Preventing School Failure, 53(4), 235-244. 
Cornejo, José. (1999). Profesores que se inician en la docencia: algunas reflexiones al respecto desde América Latina. Revista Iberoamericana de Educación, (19), 51-100.

Davini, María. (1995). Formación docente en cuestión: política y pedagogía. Buenos Aires, Argentina: Paidós.

Duschatzky, Silvia. (2001). Todo lo sólido se desvanece en el aire. En Silvia Duschatzky y Alejandra Birgin (Eds.), ¿Dónde está la escuela? (pp. 127- 145). Buenos Aires, Argentina: Manantial- Flacso.

Fernández-Cruz, Manuel. (2008). El practicum en la construcción del conocimiento profesional docente. En Enrique Correa, Gervais Colette y Sylvia Rittershaussen (Eds.), Vers une conceptualisation de la situation de stage: explorations internationals (pp. 67-89). Montreal, Canadá: Editions du CRP.

García, María y García, Juan. (2012). Filosofía de la educación. Cuestiones de hoy y de siempre. Madrid, España: UNED.

Imbernón, Francisco. (2007). La formación y el desarrollo profesional del profesorado. Hacia una nueva cultura profesional. Barcelona, España: Editorial Grao.

Ley № 20.903. Crea el Sistema de Desarrollo Profesional Docente y Modifica otras normas. (2016). Santiago, Chile: Ministerio de Educación.

Marcelo, Carlos. (2009). Los comienzos en la docencia: un profesorado con buenos principios. Profesorado. Revista de curriculum y formación del profesorado, 13(1), 1-25.

Marcelo, Carlos. (2008). El profesorado principiante. Inserción a la docencia. Barcelona, España: Octaedro.

Meirieu, Phillippe. (2003). Educar en la incertidumbre. Barcelona, España: Alertes.

Meirieu, Phillippe. (1998). Frankenstein educador. Barcelona, España: Laertes.

Pérez, Gloria. (2007). Investigación cualitativa. Madrid, España: La Muralla.

Ruffinelli, Andrea, Cisternas, Tatiana y Córdoba, Claudia. (2017). Iniciarse en la docencia. Santiago, Chile: Ediciones Universidad Alberto Hurtado.

Ruíz Olabuénaga, José. (2009). Metodología de la investigación cualitativa. Bilbao, España: Universidad de Deusto.

Tardif, Maurice, y Borges, Cecilia. (2013) La inserción profesional de docentes noveles: tendencias recientes y retos futuros. En Enrique Correa, Mónica Cividini, Rodrigo Fuentealba e Ingrid Boerr (Eds.), Formación e inserción profesional: desafíos y pistas de facilitación para la profesionalización docente (pp. 19-43). Santiago, Chile: SDL Impresores. 
Tardif, Maurice. (2004). Los saberes del docente y su desarrollo profesional. Madrid, España: Narcea.

Tenti, Emilio. (2007). Consideraciones sociológicas sobre profesionalización docente. Educação \& Sociedade, 28(99), 335-353.

Tenti, Emilio. (2004). Viejas y nuevas formas de autoridad docente. Todavía, (07), 38-43. Recuperado de http://www.revistatodavia.com.ar

Tiramonti, Guillermina. (2004). La trama de la desigualdad educativa. Buenos Aires, Argentina: Manantial.

Vélaz de Medrano, Consuelo. (2009). Competencias del profesor-mentor para el acompañamiento al profesorado principiante. Revista de currículo y formación del profesorado, 13(1), 209-299.

Wanderley, María. (2001). A Ruralidade no Brasil Moderno. Por un pacto social pelo desenvolvimento rural. En Norma Giarracca (comp), ¿Una Nueva Ruralidad en América Latina? (pp. 31-44). Buenos Aires, Argentina: CLACSO.

Zeichner, Ken. (2010). Nuevas epistemologías en formación de profesorado. Repensando las conexiones entre las asignaturas del campus y las experiencias de prácticas de formación del profesorado en la universidad. Revista Interuniversitaria de Formación del Profesorado, 68(24,2), 123-149. 